\title{
Història i Projecte Social. Reconeixement a una trajectòria - Historia y Proyecto Social. Jornadas de debate del « Institut Universitari d'Història Jaume Vicens Vives »
}

\section{Lluís Roura}

\section{OpenEdition}

\section{Journals}

Édition électronique

URL : https://journals.openedition.org/ahrf/1755

DOI : $10.4000 /$ ahrf. 1755

ISSN : 1952-403X

Éditeur :

Armand Colin, Société des études robespierristes

Édition imprimée

Date de publication : 1 juin 2004

Pagination : $244-246$

ISSN : 0003-4436

Référence électronique

Lluís Roura, «Història i Projecte Social. Reconeixement a una trajectòria - Historia y Proyecto Social. Jornadas de debate del « Institut Universitari d'Història Jaume Vicens Vives » », Annales historiques de la Révolution française [En ligne], 336 | avril-juin 2004, mis en ligne le 20 février 2006, consulté le 23 avril 2022. URL : http://journals.openedition.org/ahrf/1755 ; DOI : https://doi.org/10.4000/ahrf.1755

Ce document a été généré automatiquement le 23 avril 2022.

Tous droits réservés 


\title{
Història i Projecte Social.
} Reconeixement a una trajectòria Historia y Proyecto Social. Jornadas de debate del « Institut Universitari d'Història Jaume Vicens Vives »

\author{
Lluís Roura
}

\section{RÉFÉRENCE}

Josep Fontana, Història i Projecte Social. Reconeixement a una trajectòria, Barcelone, éd. Crítica, 2004, 2 vol., 1937 p., ISBN 84-8432-528-8.

Josep Fontana, Historia y Proyecto Social. Jornadas de debate del «Institut Universitari d'Història Jaume Vicens Vives ", Barcelone, éd. Crítica et Universitat Pompeu Fabra, 2004, 298 p., ISBN 84-8432-527-X, 20,90€.

1 Peu d'historiens ont été capables d'arriver à mériter une reconnaissance professionnelle et personnelle comme celle de Josep Fontana. On connaissait le poids de son prestige, le mérite unanimement reconnu de son œuvre et l'étendue de son magistère; mais si quelqu'un pouvait encore en douter, ces trois monumentaux volumes d'hommage viendraient lever ses doutes.

2 À l'occasion de sa retraite en 2002 l'Universitat Pompeu Fabra de Barcelone (où il a été professeur, ainsi que directeur de l'Institut d'Història Jaume Vicens Vives, au cours des dernières années et où il est actuellement professeur émérite) avait organisé quelques journées d'hommage, dont les principales conférences viennent d'être publiées chez Crítica comme un volume indépendant. On y trouve un article de Ramon Villares avec des très intéressantes réflexions autour de la "révolution libérale espagnole ». Selon Villares, la vision de la révolution libérale espagnole (un centre d'intérêt très 
remarquable dans l'historiographie espagnole, qui l'a toujours considérée comme le moment fondateur de l'Espagne contemporaine) a changé notablement au cours des trente dernières années. Mais si les études de Josep Fontana ont été fondamentales pour la vision qu'on en avait dans les années 1970 et 1980, la nouvelle vision à laquelle on est arrivé aujourd'hui selon R. Villares (par exemple les conclusions sur le poids de la modération sociale dans la révolution espagnole, qui transforme la réussite révolutionnaire en "échec »; l'importance des pouvoirs locaux et du caractère plutôt populaire de la révolution) n'est pas étrangère à son magistère. Fontana, en effet, a toujours été ouvert aux préoccupations pour la compréhension du passé dès l'engagement avec les problèmes du monde dans lequel vit l'historien. Et c'est précisément cette « ré-vision » de l'histoire de la révolution libérale qui fait l'objet des réflexions - inévitablement polémiques - de cette contribution. Les autres chapitres de ce volume concernent d'autres chantiers qui ne sont pas moins fondamentaux dans l'œuvre et le magistère de J. Fontana: F. Comín présente un état de la recherche sur l'historiographie des finances pour la période 1808-1874; A. M. Bernal fait le point sur les rapports entre le réformisme, le libre commerce, la croissance économique et les colonies américaines, entre les XVIIIe et XIXe siècles. Après le large et remarquable bilan historiographique du "problème agraire» en Espagne présenté par P. Ruiz Torres, J. Casanova nous offre un bref et incisif texte sur le changement du poids et des intérêts de l'histoire sociale dans l'historiographie espagnole; enfin, le volume se conclut avec un texte de R. López Facal incitant à la réflexion et à la discussion sur l'enseignement de l'histoire en Espagne.

3 À ce volume viennent s'ajouter les deux volumes qui recueillent la massive réponse des historiens à l'appel fait par le Département d'histoire moderne et contemporaine de l'Universitat Autònoma de Barcelona (où Fontana avait été professeur pendant une vingtaine d'années) de lui dédier un mélange d'hommage.

4 Les deux volumes mentionnés réunissent au total 145 auteurs (pour 131 textes), parmi lesquels les cinq écrits de présentation signés par les coordinateurs de l'œuvre, F. Espinet et B. de Riquer, et les professeurs Jordi Nadal, Eric Hobsbawm, John Lynch et J. Albareda. Les travaux ici réunis sont regroupés en cinq grandes parties: une partie "générale" et quatre parties chronologiques (époque moderne; de la fin XVIIIe siècle à 1868 ; 1868-1939; enfin, de 1939 à nos jours) ; chacune de celles-ci étant en même temps subdivisée en quatre grands blocs thématiques (économie, société, politique, culture). En dépit d'un nombre assez équilibré de contributions sur les respectifs champs thématiques et chronologiques, il n'est pas surprenant que globalement on puisse distinguer un nombre plus élevé de travaux sur les sujets et la période qui ont été également un objet d'attention spéciale dans la recherche et le magistère de Josep Fontana ; c'est-à-dire l'histoire économique (avec un total de 51 contributions - contre respectivement 33,24 et 18 textes consacrés aux thématiques politique, sociale et culturelle), et la période qui va de la fin du XVIIIe siècle à 1868 (avec un total de 35 contributions, à côté des 31 réservées à l'époque moderne, 27 à la période 1868-1939 et 27 autres aux années postérieures à 1939).

5 Mais ce n'est pas sur le nombre et l'ampleur des travaux ici réunis que je voudrais attirer l'attention, mais plutôt sur l'intérêt considérable et la qualité de la plupart d'entre eux, tout spécialement de ceux qui se réfèrent à la période de la crise de l'Ancien Régime et à la révolution en Espagne. Ici encore, c'est l'histoire économique qui l'emporte quant au nombre des apports (18 contributions - ou plus d'une vingtaine 
si l'on prend en compte quelques études de longue durée -, à côté de 6 écrits consacrés à la thématique sociale, 8 aux questions politiques et 3 à la culture), abordant des thématiques très diverses (les transformations du paysage et de la production agraire, l'importance particulière de divers aspects relatifs au commerce et aux colonies, les finances -municipales, provinciales et de l'État -, les transformations patrimoniales, l'industrialisation et les communications, l'importance du papier monnaie au XIXe siècle, l'évolution du coût de la vie, etc.). Pour ce qui concerne la proximité thématique avec le sujet auquel est réservé le présent numéro des A.H.R.F. - c'est-à-dire la thématique politico-culturelle et sociale de la période de la révolution libérale espagnole -, on peut remarquer des apports très significatifs autour des questions qui ont présidé aux études et débats des spécialistes tout au long des trente dernières années. D'abord, l'occupation napoléonienne: l'historien R. Fraser souligne la spécificité des attitudes et de la conduite populaire, ainsi que de leur concept de "patriotisme ", nettement différent du "patriotisme» de élites; A. Moliner, quant à lui, se réfère au mythe absolutiste du roi Fernando «El Deseado »; puis, L. Barbastro nous rappelle les négociations secrètes du traité de Valençay (décembre 1813) ; enfin, A. Gil Novales étudie le contenu au cours de l'année 1808 d'un journal d'un très remarquable intérêt pour l'étude de l'occupation napoléonienne, le Diario de Barcelona. Quelques études biographiques remettent au premier plan certains personnages d'un intérêt important et toutefois souvent oubliés - tels Isidoro de Antillón (étudié par E. Fernández) ou le baron de Savassona (étudié par M. Ramisa) - ; et R. Del Río nous propose une relecture de l'image toujours controversée d'Espoz y Mina. Pour sa part, R. Robledo nous rappelle les difficultés des libéraux au moment de la restauration absolutiste en 1814, cependant qu'A. M. García analyse celles des exilés après le Trienio liberal ; une période, sur laquelle R. Arnabat fait le point historiographique pour ce qui concerne la Catalogne. Deux questions « péninsulaires » qui ont également occupé une place importante dans la sensibilité historique de Josep Fontana centrent aussi l'attention sur deux notions : l' « ibérisme » (étudié dans ses origines par I. Castells) et le «catalanisme " (étudié par J. Pich i Mitjanase, pour la période 1859-1868). Enfin, on peut aussi mentionner la présence de la réflexion autour du caractère libéral et/ou bourgeois de la révolution espagnole, à travers les travaux écrits depuis des perspectives diverses par Jesús Millan et M. C. Romeo - du côté socio-politique - et par J. De la Torre ou encore A. Solà à partir des études socio-économiques.

6 L'édition des deux volumes d'hommage à Josep Fontana inclut la recension complète des références de l'œuvre de celui-ci (au-delà de ses 21 livres publiés jusqu'à présent, on peut trouver dans les 25 pages de cette recension plus de 500 références à ses écrits articles, chapitres de livres, préfaces, comptes rendus...). Tout un appareil historiographique stimulant pour attendre la publication de son œuvre complète, annoncée déjà par l'éditeur Crítica.

On doit donc se féliciter de l'initiative aussi bien que de l'édition menées conjointement par le Département d'histoire moderne et contemporaine de l'Université Autonome de Barcelone et l'éditeur Crítica. Ils nous permettent d'avoir, à travers ces deux volumes, un véritable aperçu de l'éventail des chantiers thématiques et chronologiques de la plus vivace et critique historiographie espagnole au début du XXIe siècle, laquelle doit précisément en grande partie son ampleur, sa vigueur et sa profondeur scientifique à l'empreinte et au magistère de Josep Fontana. 
8 On ne peut enfin pas conclure ce compte rendu sans adresser quelques reproches aux éditeurs : pourquoi n'a-t-on point inclus un index alphabétique des auteurs qui ont collaboré aux différents volumes? Pourquoi, par ailleurs, n'a-t-on pas inclus l'index général des deux volumes de mélanges dans chaque tome, et non seulement dans le deuxième? Enfin, et surtout, pourquoi n'a-t-on pas rassemblé en une seule œuvre d'hommage les trois volumes, au lieu de proposer comme une oeuvre indépendante le volume le plus mince? L'intérêt strictement commercial, que l'on peut ici deviner, est vraiment dommageable pour l'ensemble de ce travail. 